
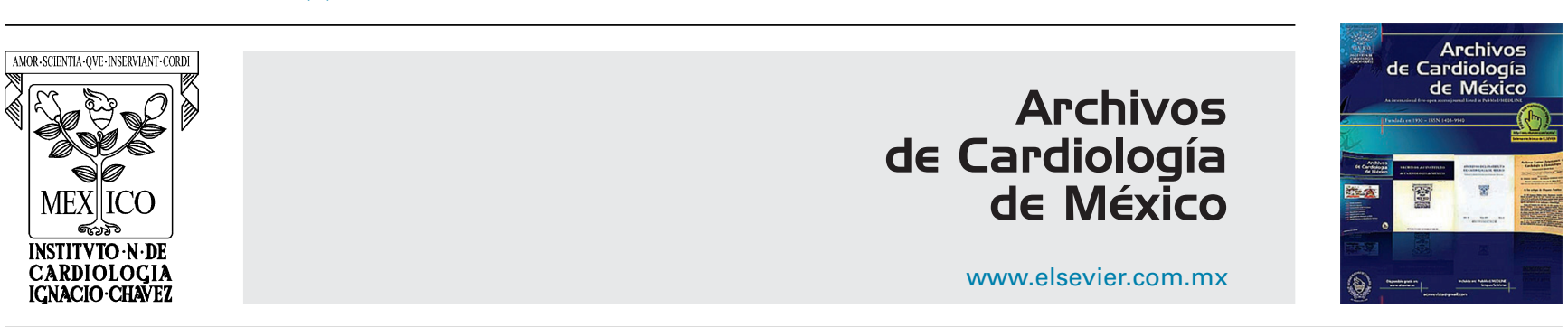

INVESTIGACIÓN BÁSICA

\title{
Situación futura de la cardiopatía isquémica en el estado de San Luis Potosí: un modelo dinámico predictivo
}

\author{
Darío Gaytán-Hernández, Aracely Díaz-Oviedo*, Verónica Gallegos-García \\ e Yolanda Terán-Figueroa
}

Facultad de Enfermería y Nutrición, Universidad Autónoma de San Luis Potosí, San Luis Potosí, México

Recibido el 19 de enero de 2017; aceptado el 25 de octubre de 2017

\author{
PALABRAS CLAVE \\ Cardiopatía \\ isquémica; \\ Factores de riesgo; \\ Análisis multivariado; \\ Modelos predictivos; \\ México
}

\begin{abstract}
Resumen
Objetivo: Desarrollar un modelo dinámico predictivo para generar y analizar la situación futura de la tasa de incidencia de la enfermedad isquémica del corazón en población de 25 años y mayores en México, en función de la variación en el tiempo de algunos factores de riesgo.

Método: Estudio ecológico retrospectivo durante el periodo 2013-2015, en la ciudad de San Luis Potosí (México). Se utilizaron bases de datos secundarias con indicadores oficiales de los 58 municipios que conforman el estado de San Luis Potosí, los cuales corresponden a los años 2000, 2005 y 2010. Se analizaron 8 indicadores a nivel municipio, por medio de los métodos de análisis de componentes principales, modelos de ecuaciones estructurales, modelaje dinámico y software de simulación.

Resultados: Fueron extraídos 3 componentes que en conjunto explican el $80.43 \%$ de la varianza total de los indicadores oficiales utilizados; el segundo componente tiene un peso de 16.36 unidades que favorecen el incremento de la enfermedad analizada; este componente está integrado solo por el indicador EDAD 60-64 y el escenario esperado del mismo va en aumento. El modelo estructural confirma que los indicadores explican el $42 \%$ de la variación de esta enfermedad; los posibles escenarios para los años 2015, 2020 y 2025 son de 195.7, 240.7 y 298, respectivamente, por cada 100,000 habitantes de 25 años y mayores.

Conclusiones: Se espera un incremento exponencial en la tasa de incidencia de la enfermedad isquémica del corazón; la edad de 60-64 años se identificó como el factor de riesgo de más peso.

(C) 2017 Instituto Nacional de Cardiología Ignacio Chávez. Publicado por Masson Doyma México S.A. Este es un artículo Open Access bajo la licencia CC BY-NC-ND (http://creativecommons. org/licenses/by-nc-nd/4.0/).
\end{abstract}

\footnotetext{
* Autor para correspondencia. Facultad de Enfermería y Nutrición. Universidad Autónoma de San Luis Potosí .Av. Niño Artillero \#130. Zona Universitaria. C. P. 78230 San Luis Potosí, México.

Correo electrónico: aracelydiaz@uaslp.mx (A. Díaz-Oviedo).
} 


\section{KEYWORDS}

Ischaemic heart

disease;

Risk factors;

Multivariate analysis;

Predictive models;

México
Future status of ischaemic heart disease in the state of San Luis Potosí: A predictive dynamic model

\begin{abstract}
Objective: To develop a predictive dynamic model to generate and analyse the future status of the incidence rate of ischaemic heart disease in a population of 25 years and over in Mexico, according to the variation in time of some risk factors.

Method: Retrospective ecological study performed during the period 2013-2015, in San Luis Potosí City, Mexico. Secondary databases that corresponded to the years 2000, 2005, and 2010, were used along with official indicators of the 58 municipalities of the state of San Luis Potosí. Eight indicators were analysed at municipality level, using principal components analysis, structural equation modelling, dynamic modelling, and simulation software methods. Results: Three components were extracted, which together explained $80.43 \%$ of the total variance of the official indicators used. The second component had a weight of 16.36 units that favoured an increase of the disease analysed. This component was integrated only by the indicator AGE 60-64 and the expected stage of it increasing. The structural model confirmed that the indicators explain $42 \%$ of the variation of this disease. The possible stages for the years 2015,2020 , and 2025 are $195.7,240.7$, and 298.0, respectively for every 100,000 inhabitants aged 25 and over.

Conclusions: An exponential increase in the incidence rate of ischaemic heart disease is expected, with the age of 60-64 years being identified as the highest risk factor.

(C) 2017 Instituto Nacional de Cardiología Ignacio Chávez. Published by Masson Doyma México S.A. This is an open access article under the CC BY-NC-ND license (http://creativecommons. org/licenses/by-nc-nd/4.0/).
\end{abstract}

\section{Introducción}

La enfermedad isquémica del corazón representa un problema de salud. En el año 2015 murieron más de ocho millones de personas por esta enfermedad en el mundo ${ }^{1}$; en México ocupó el segundo lugar como causa de mortalidad general en el periodo del 2000 al 2008, con tasas brutas ascendentes de 43.5 a 55.8 por $100,000^{2}$. Asimismo, en el año 2011 se registró una tasa de incidencia de 73.71 por cada 100,000 habitantes mayores de 14 años en el estado de San Luis Potosí ${ }^{3}$.

Esta enfermedad es multifactorial; desde los primeros estudios hasta la actualidad se han identificado más de 300 factores de riesgo, entre ellos el sedentarismo y la obesidad $^{2}$. Armas et al. en el $2009^{4}$ encontraron diferencias significativas en la prevalencia de enfermedad isquémica del corazón en población de 15 años y mayores, donde es mayor en personas con nivel de educación primaria, en población con situación económica baja y muy baja, en mujeres y también con el incremento de la edad.

De igual manera, Périssé et al. encontraron en 2010 una correlación positiva significativa en la proporción de domicilios con jefes de familia con ingresos menores o iguales a 3 salarios y las tasas de mortalidad por enfermedad isquémica del corazón y una correlación negativa con jefes de familia con salarios superiores o iguales a 15 salarios mínimos ${ }^{5}$.

Así mismo, Álvarez et al. identificaron una relación significativa de causalidad entre el sedentarismo y el infarto agudo al miocardio (RM 3.4, con intervalo 1.1-11.6) ${ }^{6}$. En otro estudio de 2012 Bacallao et al. encontraron un efecto significativo sobre la densidad de enfermos del corazón $(p<0.001)$ en un conglomerado compuesto por núcleos familiares que en promedio tienen un menor número de integrantes, más baja escolaridad, un número discretamente menor de equipos de primera necesidad y una percepción más desfavorable de su situación económica ${ }^{7}$.

Los estudios anteriores muestran la relación de los factores con la enfermedad y analizan la relación desde una perspectiva lineal, sin tener en cuenta las posibles correspondencias multivariadas de manera conjunta y simultánea entre ellos; otros autores encontraron que la presencia simultánea de varios factores de riesgo tiene un efecto multiplicativo de cada factor ${ }^{2}$. Por lo tanto, para apoyar la buena toma de decisiones es necesario el uso de modelos estadísticos que permitan analizar de manera integral las múltiples relaciones entre los factores de riesgo, así como la variación en el tiempo y el espacio que estos presentan.

Por lo anterior, el objetivo del estudio fue desarrollar un modelo dinámico predictivo para generar y analizar la situación futura de la tasa de incidencia de la enfermedad isquémica del corazón (enfermedades con clave CIE 10 I20-125) ${ }^{8}$ en población de 25 años y mayores, en función de la variación en el tiempo de algunos de los factores de riesgo, mediante una metodología consistente, considerando las relaciones complejas de los factores que la determinan, el peso que cada uno representa para la enfermedad estudiada, así como la evolución en el tiempo y el espacio de dichos factores.

\section{Métodos}

Se realizó un estudio ecológico retrospectivo durante el periodo 2013-2015 en la ciudad de San Luis Potosí (México). 
Se utilizaron bases de datos secundarias (bases de datos con información válida ya desarrolladas por otros investigadores que pueden resultar útiles para un estudio concreto) con indicadores oficiales de los 58 municipios que conforman el estado de San Luis Potosí, los cuales corresponden a los años 2000, 2005 y 2010. Estos se obtuvieron de distintas fuentes estatales y nacionales; en población $\geq 25$ años: para la tasa anual de incidencia de enfermedad isquémica del corazón por cada 100,000 habitantes $\geq 25$ años de edad $(\text { TIEIC })^{8}$, los casos de obtuvieron de los servicios de salud del estado de San Luis Potosi ${ }^{8}$ porcentaje de población femenina (FEMENINA) ${ }^{9}$, para los porcentajes de población rural (RURAL) (vive en localidades $<2,500$ habitantes) ${ }^{9}$, porcentajes de población por grupos de edad: de 45 a 49 años ${ }^{9}$, de edad 50 a $59^{9}$ y de 60 a 64 años $^{9}$ y en población general: número de automóviles registrados en circulación por 100 habitantes (AUTOS) ${ }^{10}$; estos indicadores se recuperaron del Instituto Nacional de Estadística y Geografía, y para el índice de marginación (MARGINACION) ${ }^{11,12}$ se utilizó la base del Consejo Nacional de Población. Algunos indicadores se consideraron como estimadores de los factores de riesgo, como el caso de los AUTOS para el sedentarismo y la obesidad (se utilizó por ser el único indicador oficial disponible como estimador de los factores mencionados), la MARGINACION y la población RURAL para los estilos de vida y de menos recursos.

Se tomaron como base los indicadores oficiales y con ellos se estimaron las tasas y porcentajes, excepto el índice de marginación, que se obtuvo directamente de la fuente oficial. En la construcción del índice de marginación se calcularon 10 indicadores socioeconómicos, los porcentajes de población: de 6 a 14 años que no asisten a la escuela, de 15 años o más sin educación básica completa, sin derechohabiencia a los servicios de salud; porcentaje de hijos fallecidos de las mujeres de 15 a 49 años de edad. Porcentaje de viviendas particulares habitadas: sin agua entubada dentro de la vivienda, sin drenaje conectado a la red pública o fosa séptica, sin excusado con conexión de agua, con piso de tierra, con algún nivel de hacinamiento sin refrigerador ${ }^{13}$.

La metodología integra 3 herramientas: el análisis de componentes principales, modelos de ecuaciones estructurales para confirmar las dimensiones identificadas por el análisis de componentes principales, modelaje dinámico y software de simulación para estimar escenarios futuros de la enfermedad estudiada.

En el análisis de componentes principales y los modelos de ecuaciones estructurales se utilizaron los indicadores a nivel municipio, quedando la muestra conformada por 109 registros.

Se realizó un análisis factorial exploratorio mediante el método multivariante de análisis de componentes principales para identificar componentes (variables latentes) y reducir la dimensión de los indicadores ${ }^{14}$. Se generó la tabla de la varianza total explicada para identificar el número de componentes con eigenvalores mayores de 1 , así como el porcentaje de varianza que explican ${ }^{14,15}$.También se trabajó con una matriz de componentes rotada por el método Varimax ${ }^{14,15}$. Para el procesamiento y análisis se utilizó el programa estadístico PASW Statistics versión 18. Posteriormente, con la técnica multivariante de modelos de ecuaciones estructurales, en el software Amos versión 20 se desarrolló un modelo estructural confirmatorio de medida y un modelo estructural confirmatorio de trayectorias causaefecto para identificar el peso que cada factor de riesgo tiene sobre la tasa de la enfermedad isquémica del corazón. Para ello, se tomó como base la estructura descrita por el análisis de componentes principales, 2 de los 3 componentes extraídos en dicho análisis se convirtieron en variables latentes no observables y la tasa de la enfermedad isquémica del corazón en la variable endógena.

Se validaron las estimaciones infractoras, identificándose 2 con varianza negativa, por lo que se agregaron dos restricciones y se fijaron las varianzas con un valor de $0.005^{16,17}$; a partir de esta modificación se generaron los modelos estructurales. Se utilizó la solución no estandarizada para facilitar en el modelo dinámico predictivo la interpretación del peso que cada factor de riesgo representa para la tasa de incidencia de la enfermedad isquémica del corazón. Del modelo estructural de trayectorias causa-efecto se obtuvo el porcentaje de varianza de la tasa de incidencia de la enfermedad isquémica del corazón que es explicada por el conjunto de factores de riesgo analizados (porcentaje explicado), la carga que cada componente tiene sobre la tasa de incidencia de la enfermedad isquémica del corazón (carga componente) y la que cada factor tiene en el componente (carga factor).

A continuación se desarrollaron 8 submodelos dinámicos predictivos mediante el software Vensim (uno para cada indicador), así como un modelo holístico integrado por los 8 submodelos; se utilizaron datos disponibles (valores observados) a nivel estatal; las simulaciones realizadas fueron a partir del año 2000 y hasta el 2025. El submodelo tasa de incidencia de la enfermedad isquémica del corazón fue simulado con datos de entrada provenientes de los otros 7.

Las constantes de cambio de los grupos de edad: de 0-24 (CTC0-24), de 25-44 (CTC25-44), de 45-49 (CTC45-49), de 50-59 (CTC50-59), de 60-64 (CTC60-64) y de 65+ (CTC65+) años, así como de la población rural (CTCPR), población femenina (CTCPF) y automóviles en circulación (CTCARC), se estimaron mediante la fórmula:

Constante $=[($ valor en el año final/valor en el año inicial)(1/periodo de años) $]-1$

donde, «valor en el año final» es el valor numérico que corresponde al indicador analizado en el año final del periodo considerado; «valor en el año inicial» es el valor numérico que corresponde al indicador analizado en el año inicial del mismo periodo; «periodo de años» es el número de años comprendidos en el periodo considerado ${ }^{18}$; y para el índice de marginación (CTCM) se calculó el promedio anual, tomando a todos los municipios del estado; la constante se estimó con el promedio de las diferencias anuales.

Se consideraron como valores observados datos oficiales para los años 2000, 2005 y 2010, y proyecciones propias (por el método de interpolación lineal) para los años 2001, 2002, 2003, 2004, 2006, 2007, 2008 y 2009; en los indicadores EDAD 45-49, EDAD 50-59, EDAD 60-64, RURAL y FEMENINA. Para la tasa de incidencia de la enfermedad isquémica del corazón y automóviles registrados en circulación se consideraron valores observados en todo el periodo 2000-2010; para el indicador MARGINACION, solo valores de los años 2000, 2005 y 2010.

Para las tablas de las tasas de emigración se utilizaron proyecciones del periodo 1990-2030, realizadas por el Consejo Nacional de Población ${ }^{19}$. 
Las pendientes se calcularon con los valores estimados por la simulación, con la función «pendiente» de Excel. Los submodelos dinámicos predictivos fueron validados mediante la correlación de los valores observados con los estimados por la simulación, los indicadores: EDAD 45-49, EDAD 50-59, EDAD 60-64, RURAL, FEMENINA, AUTOS y MARGINACION se validaron mediante el coeficiente de correlación de Pearson; para la tasa de incidencia de la enfermedad isquémica del corazón se realizó una validación visual mediante el gráfico de dispersión.

El efecto que cada factor tiene sobre la tasa de incidencia de la enfermedad isquémica del corazón se estimó de la siguiente manera:

1. Estimación de la proporción del peso que cada factor tiene en el componente:

Peso factor = carga factor / suma de las cargas de los factores del componente

2. Estimación de la carga que cada factor tiene sobre la tasa de incidencia de la enfermedad isquémica del corazón:

Carga factor TIEIC = peso factor ${ }^{*}$ carga componente

Los valores iniciales de cada factor son los observados en el año 2000 (valor en el año inicial).

La estimación de los efectos individuales de cada factor sobre la tasa de incidencia de la enfermedad isquémica del corazón se indica en la fórmula:

Efecto de factor $=($ factor - valor en el año inicial $){ }^{*}$ carga factor TIEIC

donde «factor» es el valor de cada indicador que se va obteniendo en la simulación.

La suma de los efectos se estimó con la siguiente fórmula:

Suma de efectos = efecto población de 4549 +efecto población de 50-59+efecto población de 60-64 + efecto población rural +efecto automóviles en circulación + efecto población femenina + efecto índice de marginación

Para estimar la tasa de incidencia de la enfermedad isquémica del corazón anual se multiplicó la suma de efectos por el porcentaje de varianza de la tasa de incidencia de la enfermedad isquémica del corazón que es explicada por el modelo estructural de trayectorias causa-efecto (VEPMCT).

Tasa de incidencia de la enfermedad isquémica del corazón = suma de efectos * VEPMCT

\section{Resultados}

Se identificó un alto nivel de colinealidad entre las variables analizadas (determinante $=0.008$ ) y una diferencia significativa con respecto a la matriz identidad $\left(\mathrm{Chi}^{2}=497.85, \mathrm{gl}=28\right.$, $\mathrm{p}<0.001)$. Con la prueba de Kaiser-Meyer-Olkin $(K M O=0.74)$ se determinó que las correlaciones son adecuadas para aplicar el análisis de componentes principales. Se extrajeron 3 componentes que explican el $80.43 \%$ de la varianza acumulada del conjunto de datos.

Se muestra la matriz de componentes rotados (tabla 1) por el método Varimax, que describe claramente las saturaciones de los indicadores en cada uno de los 3 componentes extraídos. El primer componente quedó conformado por 5 indicadores que en conjunto explican el $48.33 \%$ de la varianza total del conjunto de indicadores analizados, el segundo con un indicador que explica el $17.87 \%$ y el tercero con 2 indicadores que explican el $14.23 \%$ de varianza total.
Tabla 1 Matriz de componentes rotados por el método Varimax del análisis de componentes principales. San Luis Potosí (México), 2013-2015

\begin{tabular}{lrcc}
\hline Clave del indicador & \multicolumn{3}{c}{ Componente } \\
\cline { 2 - 4 } & \multicolumn{1}{c}{1} & 2 & 3 \\
\hline Marginación & -0.808 & & \\
TIEIC & 0.797 & & \\
Rural & -0.771 & & \\
Femenina & 0.745 & & \\
Autos & 0.697 & & 0.948 \\
Edad 60-64 & & 0.913 & 0.660 \\
Edad 45-49 & & & \\
Edad 50-59 & & & \\
Fuente: Elaboración propia.
\end{tabular}

Fuente: Elaboración propia.

Se muestra el modelo estructural confirmatorio de medida (fig. 1) que se diseñó a partir de los resultados del análisis de componentes principales. Los resultados se muestran en unidades no estándar; los pesos de regresión de los errores de medición y de los indicadores MARGINACION, AUTOS y EDAD 45-49 años se fijaron en 1.

Todos los estimados de los pesos de regresión son significativos $(C R>1.96$ y $p<0.001)$, así como las covarianzas entre el primer componente y el segundo y entre el segundo y el tercero $(p<0.05)$.

Los resultados estimados en unidades no estándar (fig. 2), los indicadores del primero, tercero y el indicador del segundo componente, tomados en conjunto, representan factores de riesgo para la tasa de incidencia de la enfermedad isquémica del corazón, considerando las sinergias entre los indicadores correspondientes en cada componente y sus respectivos errores de medición. Por cada incremento de una unidad del primer componente, la tasa de incidencia de la enfermedad isquémica del corazón mostrará un incremento de 5.66; asimismo, por cada incremento en una unidad del segundo componente, la tasa de incidencia de la enfermedad isquémica del corazón presentará un incremento de 16.36 y por cada unidad que incremente el tercer componente la tasa de incidencia de la enfermedad isquémica del corazón incrementará 3.46 unidades. Todos los estimados individuales de los pesos de regresión de los indicadores son significativos $(C R>1,96$ y $p<0,005)$. Sin embargo, la carga del tercer componente sobre la tasa de incidencia de la enfermedad isquémica del corazón no es significativa $(p=0.421)$.

La jerarquía de los factores de riesgo, considerando el peso que tienen en el interior del componente y la carga que el componente representa para la tasa de incidencia de la enfermedad isquémica del corazón, es: EDAD 60-64, EDAD 50-59, AUTOS, EDAD 45-49 y FEMENINA, mientras que RURAL y MARGINACION son factores protectores. Finalmente, el modelo estructural mostró que los predictores de la tasa de incidencia de la enfermedad isquémica del corazón considerados en este análisis explican el $42 \%$ de su variabilidad.

El modelo dinámico predictivo (fig. 3) muestra los flujos de cada indicador (población de 45-49, población de 50-59, población de 60-64, población rural, población femenina, automóviles en circulación e índice de marginación) hacia su respectivo efecto y estos hacia la suma de efectos para 


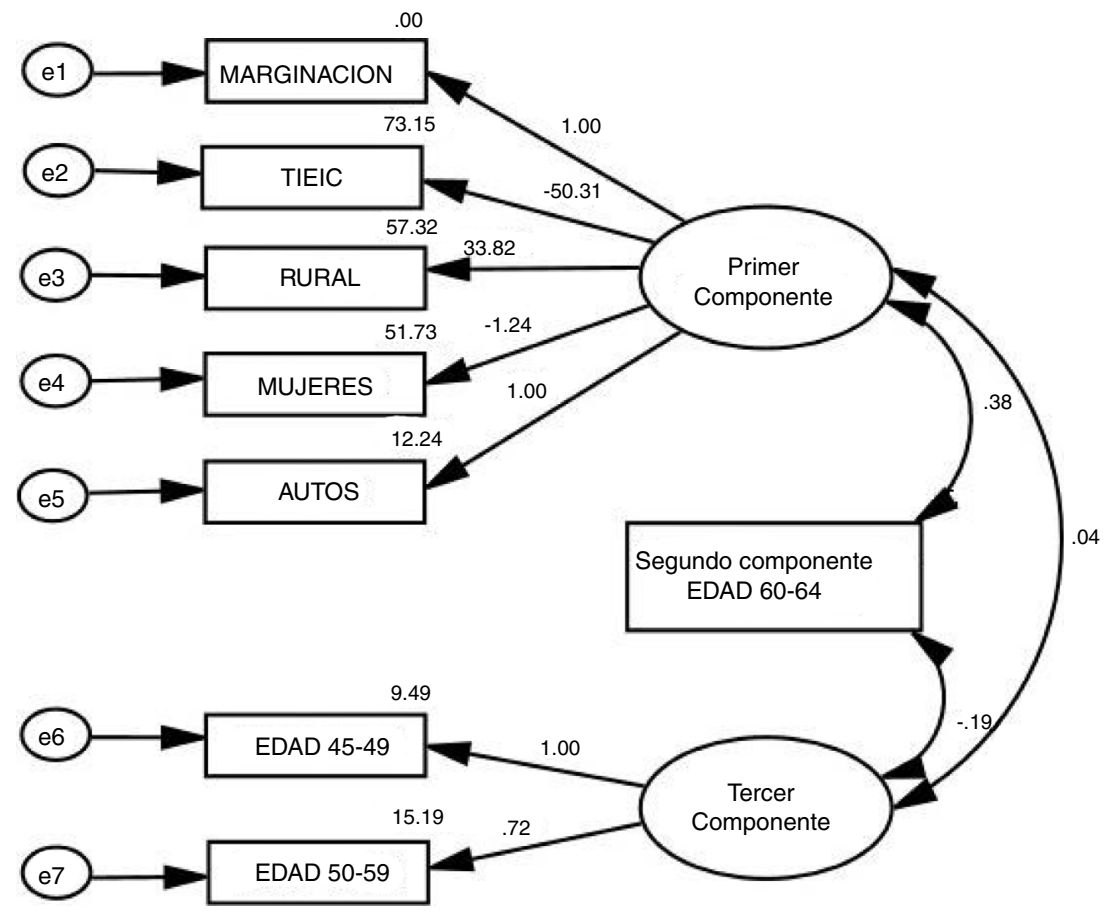

Figura 1 Modelo estructural confirmatorio de medición de las relaciones multivariadas entre la tasa de incidencia de enfermedad isquémica del corazón y algunos de sus factores de riesgo. San Luis Potosí (México), 2013-2015.

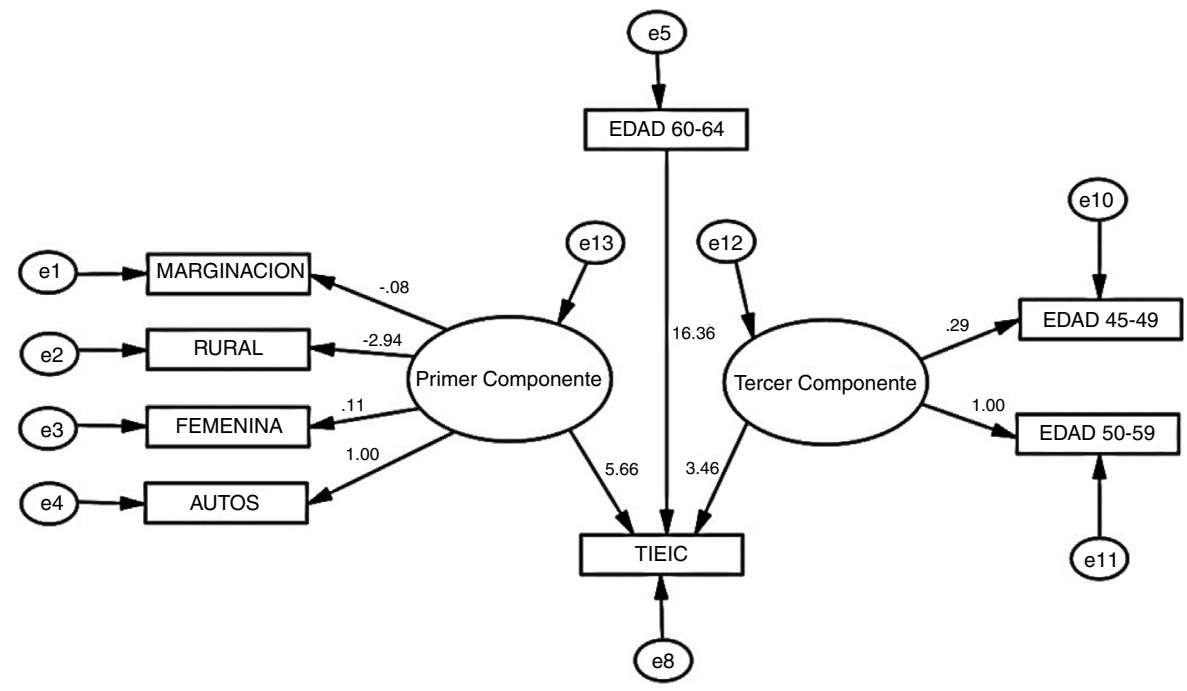

Figura 2 Modelo estructural de trayectorias causa-efecto de las relaciones multivariadas entre la tasa de incidencia de enfermedad isquémica del corazón y algunos de sus factores de riesgo. San Luis Potosí (México), 2013-2015.

que, finalmente, este flujo impacte en la tasa de incidencia de enfermedad isquémica del corazón.

En 3 de los indicadores analizados se espera un decremento; el que más tiende a bajar es EDAD 45-49 años, mientras que 5 muestran un incremento en el periodo analizado; de los factores de riesgo, el indicador AUTOS es el de mayor crecimiento. Asimismo, en la tasa de incidencia de la enfermedad isquémica del corazón, que es el punto medular de este análisis, se espera un crecimiento exponencial. Este resultado no indica que se tenga la certeza de que los escenarios van a suceder, más bien es una aproximación a una realidad de este grave problema de salud (tabla 2 ).
Los submodelos de los indicadores EDAD 50-59, EDAD 45-49, AUTOS y MARGINACION mostraron un buen ajuste, con unos coeficientes de correlación de 0.98, 0.95, 0.98 y 0.91 , respectivamente. El modelo dinámico predictivo permitió analizar la situación futura de la tasa de incidencia de la enfermedad isquémica del corazón.

\section{Discusión}

Cada componente representa un factor de riesgo para la tasa de incidencia de la enfermedad isquémica del cora- 


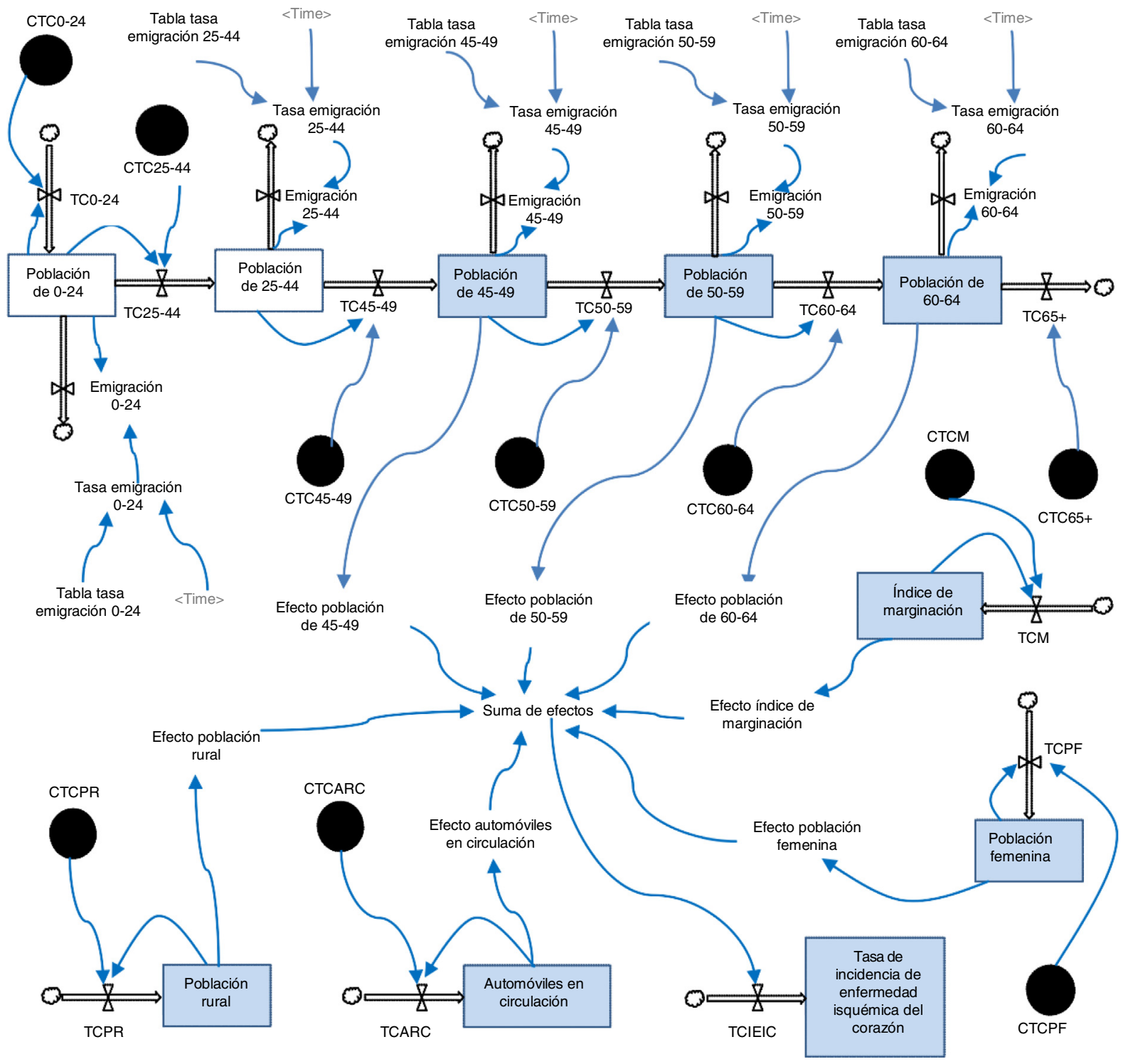

Figura 3 Modelo dinámico predictivo con enfoque holístico de la tasa de incidencia de enfermedad isquémica del corazón y algunos de sus factores de riesgo. San Luis Potosí (México), 2013-2015.

Tabla 2 Escenarios futuros probables de los indicadores. San Luis Potosí (México), 2013-2015

\begin{tabular}{lcccrrrr}
\hline Clave & 2000 & 2005 & 2010 & 2015 & 2020 & 2025 & Pendiente \\
\hline TIEIC & 122.8 & 128.5 & 157.4 & 195.7 & 240.7 & 298.0 & 7.3 \\
Edad 60-64 & 6.8 & 7.6 & 8.0 & 8.1 & 8.1 & 8.3 & 0.0488 \\
Edad 50-59 & 16.5 & 16.3 & 15.0 & 13.8 & 12.7 & 12.2 & -0.2082 \\
Edad 45-49 & 10.7 & 7.9 & 6.2 & 5.2 & 4.8 & 4.5 & -0.2242 \\
Rural & 7.4 & 7.6 & 7.8 & 7.9 & 8.1 & 8.3 & 0.0345 \\
Autos & 20.5 & 23.7 & 27.5 & 31.9 & 36.9 & 42.8 & 0.8878 \\
Femenina & 61.6 & 62.0 & 62.5 & 62.9 & 63.4 & 63.9 & 0.0914 \\
Marginación & 0.189 & 0.156 & 0.129 & 0.107 & 0.088 & 0.073 & -0.0046 \\
\hline
\end{tabular}

Fuente: Elaboración propia. 
zón; el primer componente representa un peso de 5.66 sobre la tasa de incidencia de la enfermedad isquémica del corazón, el indicador EDAD 60-64, 16.36 y el tercer componente, 3.46 unidades no estándar.

Aunque ya se han reportado como factores de riesgo de manera individual los indicadores que integran cada componente, la presencia simultánea de varios factores de riesgo tiene un efecto no solo aditivo, sino multiplicativo del riesgo de cada factor por separado ${ }^{2}$.

Por otra parte, los factores de riesgo considerados en este estudio tienen una variación en el tiempo y el espacio; los resultados de los submodelos dinámicos individuales, por sí solos, muestran probables escenarios futuros que pueden apoyar la toma de decisiones.

El escenario del indicador EDAD 60-64, el cual fue identificado como el principal factor de riesgo, muestra un incremento de $0.8 \%$ en el año 2000 y de $8.3 \%$ en el 2025 . Los escenarios del segundo y cuarto factor de riesgo en jerarquía (EDAD 50-59 y EDAD 45-49) muestran una tendencia a la baja de 16.5 a $12.2 \%$ y de 10.7 a $4.5 \%$; la jerarquía de estos factores de riesgo es congruente con el estudio realizado por Armas et al., quienes reportaron un incremento en las tasas de enfermedad isquémica del corazón con el incremento de la edad en grupos ${ }^{4}$.

El tercer factor jerárquico, AUTOS, es el que mayor incremento presenta, al pasar de 20.5 a 42.8 automóviles por cada 100 habitantes de 25 años o más; este indicador se utilizó para estimar de manera indirecta la obesidad y el sedentarismo, pues se ha identificado que el uso del automóvil es un factor para padecer obesidad ${ }^{20}$; por otro lado, la obesidad es un factor relacionado con la enfermedad isquémica del corazón ${ }^{2}$, al igual que el sedentarismo ${ }^{6}$.

En el quinto factor de riesgo, FEMENINA, se vislumbra un pequeño incremento-de 61.6 a $63.9 \%$-que puede considerarse no significativo; sin embargo, Armas et al. lo identificaron como un factor de riesgo también, pues encontraron una tasa más alta de enfermedad isquémica del corazón en mujeres, aunque analizaron una población mayor de 15 años, y en este estudio la población fue de 25 años o más ${ }^{4}$.

Por otro lado, se identificaron como factores protectores la población rural (RURAL) y el índice de marginación (MARGINACION); este resultado puede tener sentido porque las zonas rurales son las más marginadas ${ }^{21}$, por ello, se esperaría que represente un factor de riesgo de acuerdo con lo reportado por Armas et al., Périssé et al. y Bacallao et al., quienes identificaron aspectos marginales como factores de riesgo de la enfermedad isquémica del corazón ${ }^{5,7}$. Sin embargo, en el medio rural los estilos de vida son más saludables y el gasto energético es mayor que en el urbano ${ }^{22}$.

El escenario esperado de la población rural es de crecimiento de 7.4 a $8.3 \%$, el cual puede ser alentador tomando en cuenta que el incremento de esta población significa una disminución en la tasa de incidencia de la enfermedad isquémica del corazón, mientras que el escenario del índice de marginación se espera a la baja de 0.189 a 0.073 .

Finalmente, los escenarios de la tasa de incidencia de la enfermedad isquémica del corazón muestran un crecimiento exponencial (de 122.8 en el año 2000 a 298 en 2025); esto es el resultado de la interacción de los factores analizados, considerando el peso que cada uno de ellos representa para la tasa de incidencia de la enfermedad isquémica del corazón, mismo que fue estimado por el modelo estructural de trayectorias causa-efecto, así como la variación de los mismos en el tiempo (tabla 2).

Como ya se mencionó, este resultado no indica que se tenga la certeza de que los escenarios van a suceder, más bien es una aproximación a una posible realidad de este grave problema de salud en el Estado de San Luis Potosí, en el que resalta que el orden jerárquico de los factores de riesgo que afectan a la tasa de incidencia de la enfermedad isquémica del corazón es: EDAD 60-64, EDAD 50-59, AUTOS, EDAD 45-49 y FEMENINA.

\section{Conclusiones}

Los 3 métodos utilizados pueden integrarse para realizar un análisis multivariantes a diferentes niveles de profundidad: exploratorio, confirmatorio y desarrollo de modelos dinámicos holísticos predictivos. El modelo dinámico predictivo holístico muestra un escenario con un incremento exponencial alarmante en la tasa de incidencia de la enfermedad isquémica del corazón en San Luis Potosí; por otro lado, los indicadores EDAD 60-64 y EDAD 50-59 se identificaron como los factores de riesgo más determinantes de la tasa de incidencia de la enfermedad isquémica del corazón con escenarios de incremento, por lo cual, las acciones de los tomadores de decisiones estarían dirigidas principalmente hacia este grupo de edad y la disminución del uso de automóvil.

\section{Financiación}

No se recibió patrocinio de ningún tipo para llevar a cabo este artículo.

\section{Conflicto de intereses}

Los autores declaran no tener ningún conflicto de intereses.

\section{Bibliografía}

1. Organización Mundial de la Salud. Centro de prensa. Las 10 principales causas de defunción. Agosto 27, 2014 [consultado 30 Nov 2017]. Disponible en: http://www.who.int/mediacentre/ factsheets/fs310/es/index2.html

2. González R, Alcalá J. Enfermedad isquémica del corazón, epidemiología y prevención. FACMED. 2010;53:35-43.

3. Dirección General de Epidemiología. Sistema Nacional de Vigilancia Epidemiológica. 2011. Información Epidemiológica de Morbilidad. Anuario 2011 [consultado 30 Ago 2014]. Disponible en: http://www.epidemiologia.salud.gob.mx/ doctos/infoepid/publicaciones/2012/ver_ejecutiva_2011.pdf

4. Armas N, Dueñas A, de la Nova R, et al. Heart diseases and its epidemiologic features in Cuban population aged 15 and more. Revista Cubana de Investigaciones Biomédicas. 2009;28:1-13.

5. Périssé G, de Andrade R, Escosteguy CC. Espacio urbano y la mortalidad por enfermedad isquémica del corazón en adultos mayores en Rio de Janeiro. Arq Bras Cardiol. 2010;94:447-55.

6. Álvarez JT, Bello V, Pérez G, et al. Coronary risk factors associated with the acute myocardial infarction in the elderly. Medisan. 2013;17:54-60.

7. Bacallao J, Díaz-Perera G, Alemañy E. Patrones de concentración social de factores de riesgo aterosclerótico y 
enfermedades del corazón en La Habana. Rev Cubana Salud Publica. 2012;38:511-24.

8. Servicios de Salud del estado de San Luis Potosí. 2013. Sistema Único de Información para la Vigilancia Epidemiológica (SUIVE) 2007 [consultado 14 Ago 2013].

9. Instituto Nacional de Estadística y Geografía (INEGI), 2013. Censos y conteos de población y vivienda. Serie histórica censal e intercensal [consultado $28 \mathrm{Sep}$ 2014]. Disponible en: http:// www. inegi.org. $\mathrm{mx} /$ est / lista_cubos / consulta.aspx?.p=pob\&c=6

10. Instituto Nacional de Estadística y Geografía [Internet]. Registros administrativos. Vehículos de motor registrados en circulación [citado 24 May 2013]. INEGI; 2011. Disponible en: http://www.inegi.org.mx/est/contenidos/Proyectos/registros/ economicas/vehiculos/descripciones.aspx\#.

11. Consejo Nacional de Población (CONAPO). Índices de marginación 2005 [consultado 1 May 2013]. Disponible en: http:// www.conapo.gob.mx/es/CONAPO/Indices_de_marginacion_2005_

12. Consejo Nacional de Población (CONAPO). Índice de marginación por entidad federativa y municipio 2010 [consultado 1 May 2013]. Disponible en: http://www.conapo.gob.mx/es/ CONAPO/Indices_de_Marginacion_2010_por_entidad_federativa_ y_municipio

13. Consejo Nacional de Población (CONAPO). Índice de marginación urbana, 2010. Anexo C. Metodología de estimación del índice de marginación urbana 2010. Disponible en: http://conapo.gob.mx/work/models/CONAPO/Resource/862/ 4/images/06_C_AGEB.pdf.
14. Meyers LS, Gamst G, Guarino AJ. Applied multivariante research. Design and interpretation. Thousand Oaks, California: SAGE Publications; 2006.

15. Hair J, Anderson R, Tatham R, et al. Análisis multivariante. Madrid: Prentice-Hall; 2007.

16. Bentler PM, Chou C. Practical issues in structural modeling. Sociol Methods Res. 1987;16:78-117.

17. Dillon W, Kumar A, Mulani N. Offending estimates in covariance structure analysis: Comments on the causes and solutions to Heywood cases. Psychol Bull. 1987;101:126-35.

18. Gavira Durón N. Cálculo diferencial e integral con aplicaciones a la economía, demografía y seguros. [Internet] [citado 24 May 2015]. Disponible en: http://www.dynamics. unam.edu/NotasVarias/Actuarial.pdf

19. Consejo Nacional de Población (CONAPO). 2014. Proyecciones de la población 2010-2050 [consultado 27 Jul 2015]. Disponible en: http://www.conapo.gob.mx/es/CONAPO/ Proyecciones_Datos

20. Jacobson SH, King DM, Yuan R. A note on the relationship between obesity and driving. Transp Policy (Oxf). 2011;18:772-6.

21. Consejo Nacional de Población (CONAPO). 2012. Índice de marginación por localidad 2010 [consultado 14 May 2016]. Disponible en: http://www.conapo.gob.mx/en/CONAPO/ Indice_de_Marginacion_por_Localidad_2010

22. Colunga L. Obesidad y sedentarismo en poblaciones rural y urbana [tesis de maestría]. México: Universidad Autónoma de Nuevo León; 2005. 